\title{
Hallucination Severity
}

National Cancer Institute

\section{Source}

National Cancer Institute. Hallucination Severity. NCI Thesaurus. Code C156239.

An indication of the type of a hallucination and its impact on a subject. 